\title{
Psychopathological Networks in Cognitive-Behavioral Treatments for Binge-Eating Disorder
}

\author{
Anja Hilbert $^{a}$ Stephan Herpertz ${ }^{b}$ Stephan Zipfelc ${ }^{c}$ Brunna Tuschen-Caffier ${ }^{d}$ \\ Hans-Christoph Friederich ${ }^{e}$ Andreas Mayr ${ }^{f}$ Martina de Zwaan ${ }^{g}$ \\ antegrated Research and Treatment Center AdiposityDiseases, Behavioral Medicine Research Unit, Department of \\ Psychosomatic Medicine and Psychotherapy, University of Leipzig Medical Center, Leipzig, Germany; ${ }^{b}$ Department

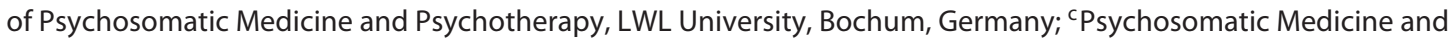 \\ Psychotherapy, Medical University Hospital Tuebingen, Tuebingen, Germany; ${ }^{d}$ Department of Clinical Psychology \\ and Psychotherapy, University of Freiburg, Freiburg, Germany; ${ }^{\text {Department }}$ of General Internal Medicine and \\ Psychosomatics, Medical University Hospital Heidelberg, Heidelberg, Germany; ${ }^{\mathrm{f}}$ Department of Medical Biometry, \\ Informatics and Epidemiology, University Hospital Bonn, Bonn, Germany; ${ }^{9}$ Department of Psychosomatic Medicine \\ and Psychotherapy, Hannover Medical School, Hannover, Germany
}

\section{Keywords}

Binge-eating disorder - Eating disorder .

Cognitive-behavioral therapy · Network analysis •

Prediction

\begin{abstract}
Introduction: Network approaches to psychopathology posit that mental disorders emerge from interrelated symptoms, and thus connectivity among symptoms are assumed to negatively predict the treatment response and decrease with efficacious treatment. Objective: This study uniquely sought to elucidate the network structure, its change, and its predictive value in cognitive-behavioral therapy (CBT) for binge-eating disorder (BED). Methods: In a multicenter randomized trial of face-to-face and Internet-based guided selfhelp CBT, 178 individuals with full syndrome and subsyndromal BED, eating disorder and general psychopathology, and body mass index (BMI) were subjected to Gaussian Graphical Network and Exploratory Graph Analyses before and after
\end{abstract}

karger@karger.com www.karger.com/pps

Karger $\frac{1}{6}$

GOPEN ACCESS
(C) 2020 The Author(s)

Published by S. Karger AG, Basel

This is an Open Access article licensed under the Creative Commons Attribution-NonCommercial-4.0 International License (CC BY-NC) (http://www.karger.com/Services/OpenAccessLicense), applicable to the online version of the article only. Usage and distribution for commercial purposes requires written permission. treatment and at 6-month follow-up. Results: At pretreatment, 3 network communities of: eating disorder psychopathology; general psychopathology; and restraint and BMI were identified, with the latter community included in the first thereafter. Eating disorder-related impairment and selfesteem were the most central symptoms, while BMI and binge eating had the lowest centrality. Network connectivity significantly increased from pre- to posttreatment, with the greatest increases in strength centrality found in binge eating and shape concern, but it did not predict remission from binge eating. Conclusions: With decreasing symptom severity, CBT resulted in a greater integration and connectivity of the psychopathology network in BED, suggesting an increased patient understanding of relations between binge eating and other symptoms. Network connectivity was not a negative prognostic indicator of treatment outcome. These results indicate a need for further research on the predictive value of network variables in the explanation of therapeutic change for patients with BED.

(C) 2020 The Author(s)

Published by S. Karger AG, Basel 


\section{Introduction}

Binge-eating disorder (BED) is characterized by recurrent binge eating in the absence of inappropriate compensatory behaviors [1]. This prevalent clinical eating disorder co-occurs with eating disorder and general psychopathology, obesity (BMI $\geq 30.0 \mathrm{~kg} / \mathrm{m}^{2}$ ), and psychosocial impairment. Cognitive-behavioral therapy (CBT) is the most well-established therapy for BED, with similar efficacy in face-to-face and CBT structured self-help treatment formats $[2,3]$, but pretreatment predictors of treatment outcomes have proven elusive [4]. Using a network approach to psychopathology, this study uniquely sought to elucidate the network structure, its change, and the predictive value in CBT for BED.

Network approaches to psychopathology posit that mental disorders emerge from a dynamic interplay among symptoms, which can be modeled using network analysis $[5,6]$ and may predict treatment outcome. In psychopathological networks, symptoms represent nodes that are connected with edges, depicting the strength and direction of correlations between symptoms. Symptoms with stronger relations to other symptoms are considered to be central to the network (i.e., have a high strength centrality). Thus, if the relations between symptoms are strong, the network connectivity is high and the network is assumed to be pathological and self-sustaining. Network theory further proposes that treatments may change single symptoms or symptom networks, depending on their efficacy [6]. If a treatment is efficacious, network connectivity was predicted to decrease after treatment [7] - an assumption that has received mixed support in mental disorders including depression [8-10]. Overall, the value of network indicators to predict treatment outcome requires further research $[11,12]$.

Eating disorder psychopathology networks $[13,14]$ cross-sectionally identified shape and weight concern, especially overvaluation of shape, as highly central in treatment-seeking individuals with BED, while binge eating and restraint were less central, and BMI had a low centrality $[15,16]$. Eating disorder and general psychopathology variables seemed to represent distinct, but interrelated, clusters in BED, with depression showing the greatest strength of associations with eating disorder psychopathology [16]. In contrast, dispositional variables such as novelty or reward seeking seemed to be less linked to eating disorder psychopathology. Prospectively, in mixed eating disorders, connectivity of the eating disorder network at admission did not change over unstandardized treatment, but it predicted less improvement at discharge
[17]. Based on this initial support for a predictive value of psychopathological network parameters, this study sought to prospectively investigate psychopathology networks in CBT for BED at pre- and posttreatment and at 6-month follow-up, hypothesizing: (1) a distinct eating disorder psychopathology community with a low integration of binge eating, restraint, and BMI; (2) unchanged network connectivity following CBT; and (3) the ability to predict remission from lower pretreatment network connectivity.

\section{Materials and Methods}

In a multicenter randomized trial of face-to-face and Internetbased guided self-help CBT [18], 178 individuals with full syndrome and subsyndromal BED (DSM-IV-TR) [19] aged $\geq 18$ years and with a BMI of $27.0-40.0 \mathrm{~kg} / \mathrm{m}^{2}$ were treated with twenty 50 min individual sessions of manualized CBT [20] or Internet-based guided self-help [21], both delivered over 4 months. For methodological detail, see the online supplementary material (for all online suppl.material, seewww.karger.com/doi/19.1159/000509458).

The treatment response at posttreatment (after 4 months of treatment) and at 6-month follow-up was defined as full remission from binge eating (i.e., zero objective binge-eating episodes [OBEs] over the past 28 days), as assessed through the clinical interview Eating Disorder Examination (EDE) [22, 23]. For network analysis, the following interview- and questionnaire-based variables were used: eating disorder psychopathology, operationalized through the number of OBEs over the past 28 days, and restraint and eating, shape, and weight concern (EDE) [22]; emotional eating (Dutch Eating Behavior Questionnaire; DEBQ) [24, 25]; eating disorder-related impairment (Clinical Impairment Assessment; CIA) [26]; and BMI, calculated from measured height and weight $\left(\mathrm{kg} / \mathrm{m}^{2}\right)$. General psychopathology was operationalized through depression (Beck Depression Inventory-II; BDI-II) $[27,28]$ and low self-esteem (Rosenberg Self-Esteem Scale; RSES) [29, 30], and temperament was operationalized through punishment and reward sensitivity (Behavioral Inhibition System/Behavioral Activation System Scales; BIS/BAS) [31, 32].

\section{Data Analytic Plan}

For treatment of missing data by multiple imputation, comparison of completers versus noncompleters, and therapeutic change in the network variables, see the online supplementary material. Each network included the 12 indicators of eating disorder psychopathology, BMI, general psychopathology, and punishment and reward sensitivity at pretreatment, posttreatment, and at 6-month follow-up. First, in order to examine how the network nodes cluster together, an Exploratory Graph Analysis [33, 34] was conducted. This analysis estimates communities in networks via a random walk algorithm (walktrap) in order to evaluate whether nodes that cluster together in communities are part of the same latent variable or dimension. In order to examine network structure at pretreatment, posttreatment, and at 6-month follow-up, Gaussian Graphical Network Analyses with LASSO (least absolute shrinkage and selection operator) regularization were used to limit the number of spurious connections in the network [35]. To explore network stability, the Bootstrapped Difference Test was conducted (bootnet 


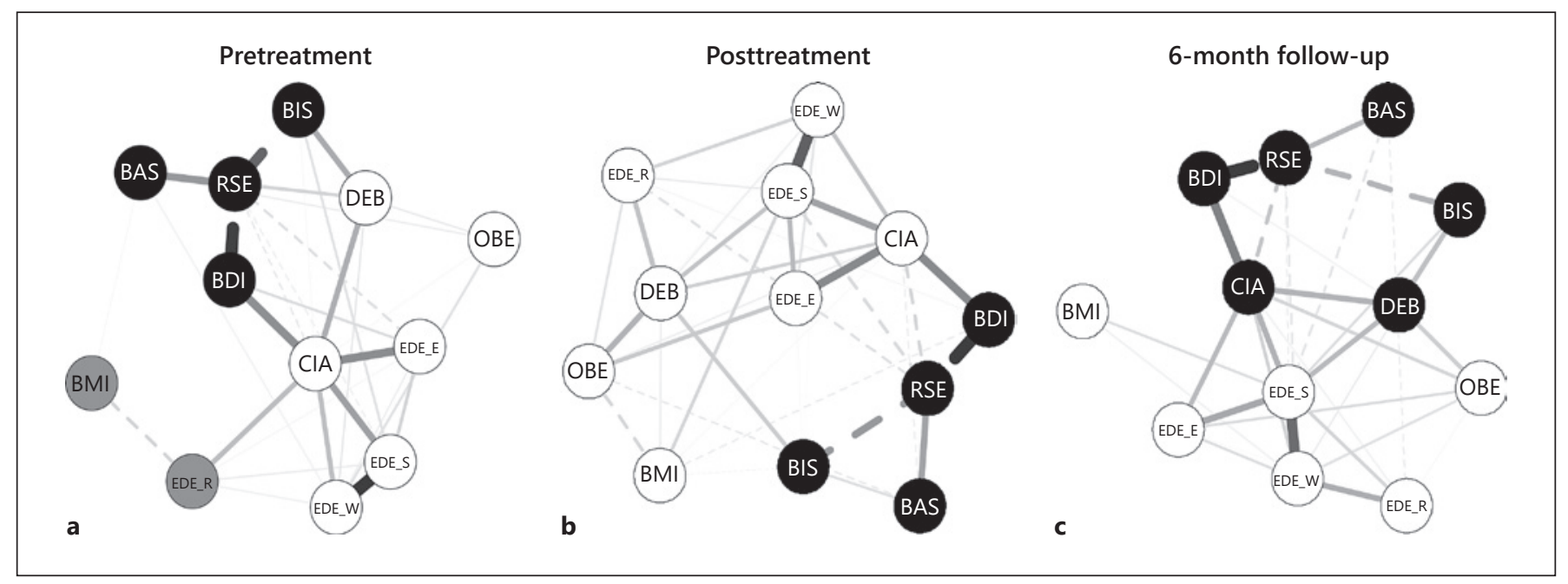

Fig. 1. Network structure of eating disorder and general psychopathology at pretreatment (a), at posttreatment (b), and at 6-month follow-up (c). Solid edges, positive associations; dashed edges, negative associations; thicker edges, stronger associations. EDE_R, dietary restraint; EDE_E, eating concern; EDE_W, weight concern; EDE_S, shape concern (all indicators from the Eating Disorder Examination); DEB, emotional eating (Dutch Eating Behavior

package) [36], and the local centrality measures of strength, closeness, and betweenness were estimated to characterize network structure (for definitions, see the online suppl. material).

Second, the change in network connectivity between pretreatment and posttreatment, as well as 6-month follow-up, was analyzed using the Network Comparison Test (NCT) for repeated measurements, including the Global Strength Invariance Test [37]. Third, for predictor analysis, we compared the pretreatment network connectivity of responders with full remission from OBEs at posttreatment and at 6-month follow-up versus that of nonresponders using between-group NCT analyses as described above.

\section{Results}

Exploratory Graph Analysis documented the following 3 pretreatment communities (Fig. 1): restraint and BMI, eating disorder psychopathology (binge eating; eating, weight, and shape concern; emotional eating; and eating disorder-related impairment), and general psychopathology (depression, self-esteem, reward and punishment sensitivity). At posttreatment and at follow-up, 2 communities were identified: restraint and BMI were included in the eating disorder psychopathology community, and the general psychopathology community remained the same as at pretreatment, with the exception
Questionnaire); CIA, eating disorder-related impairment (Clinical Impairment Assessment); BDI, depression (Beck Depression Inventory-II); RSE, self-esteem (Rosenberg Self-Esteem Scale); BIS, dispositional sensitivity to punishment (Behavioral Inhibition System Scale); BAS, dispositional sensitivity to reward (Behavioral Activation System Scale).

of eating disorder-related impairment and emotional eating as part of the latter community at follow-up.

Stability analyses for the networks at pretreatment, posttreatment, and at 6-month follow-up revealed adequate correlation stability of strength $(0.753,0.674$, and 0.674 ) and betweenness $(0.438,0.438$, and 0.281$)$. Before treatment, the Gaussian Graphical Network Analyses with LASSO regularization (Figure S1) showed the highest node strength for eating disorder-related impairment (1.75) and self-esteem (1.52), followed by shape concern (0.65). As predicted, the lowest strength was found for BMI (-1.43) and binge eating (-1.31). Eating disorderrelated impairment had the highest betweenness (2.55) and bridged between the eating disorder psychopathology and general psychopathology communities, and the restraint and BMI community.

According to the NCT's Global Strength Invariance Test, treatment resulted in a significant increase in network connectivity from pre- to posttreatment (4.10 vs. $4.79, S=0.69, p=0.02)$ but not from pretreatment to follow-up (4.10 vs. $4.37, S=0.27, p=0.25$ ). The greatest changes in local centrality from pre- to posttreatment (and 6-month follow-up) included an increase in node strength in shape concern $(0.65,1.32$, and 1.58$)$ and binge eating $(-1.31,-0.51$, and -0.77$)$ and a decrease in punishment sensitivity $(-0.05,-0.77$, and -0.59$)$. The greatest 
changes in betweenness from pre- to posttreatment (and 6-month follow-up) were an increase in shape concern $(0.52,1.28$, and 1.79$)$ and a decrease in restraint $(0.09$, -0.90 , and -0.83$)$ and eating-disorder-related impairment $(2.55,1.66$, and 1.48).

Examining network connectivity as a predictor of treatment response, the NCT's Global Strength Invariance Test did not reveal any significant differences in pretreatment network connectivity in responders with remission from binge eating versus nonresponders at posttreatment $(S=0.12, p=0.88)$ and at 6-month follow-up $(S=0.36, p=0.79)$. A sensitivity analysis using averaged weight and shape concern showed mostly consistent results, with few deviations, and a further supplementary analysis did not provide evidence for a predictive power of single nodes or local centrality indices (see online suppl. material).

\section{Discussion}

This study uniquely explored the network structure and the change and predictive value of network connectivity in a large multicenter randomized trial of CBT for individuals with full syndrome and subsyndromal BED. At pretreatment, 3 distinct network communities of eating disorder psychopathology, general psychopathology (also including dispositional sensitivity to punishment and reward), and restraint and BMI were identified, which is consistent with cross-sectional network research on BED, mixed eating disorders, and obesity [15-17, 38]. In line with most literature on these disorders [15-17, 39-42], eating disorder-related impairment, low self-esteem, and shape concern were among the most central indicators, whereas BMI and binge eating were the least central. The relatively low centrality of punishment and reward sensitivity is consistent with previous evidence on mixed eating disorders [16], likely associated with their general, non-eating-disorder-related formulation. Impairment served as a bridge symptom linking the communities (especially with depression), supporting that eating disorder-related burden was the most prevailing and eradiating symptom with which treatment-seeking individuals with BED presented.

With decreasing symptom severity, CBT for BED resulted in a greater integration of network structure, with the restraint and BMI community included in the eating disorder psychopathology community at posttreatment and at 6-month follow-up. We used Exploratory Graph Analysis [33, 34], linking network analysis with latent variable analysis in order to identify latent variables or dimensions of nodes clustering together, an approach that seeks to reconcile network and "common cause" models [43]. Following CBT, weight pathology did not form an underlying dimension distinct from eating disorder psychopathology anymore, suggesting that the respective symptoms were more closely linked in the maintenance of BED, likely following an integrated developmental pathway [44].

As opposed to the prediction of network theory, assuming that an efficacious treatment should lead to a decrease in network connectivity and its self-sustaining character [4], CBT for BED resulted in a higher connectivity of the psychopathology network, which is consistent with some $[8,9]$ but not all studies [10] on diverse mental disorders including depression. In mixed eating disorders, Smith et al. [17] did not find a change in network connectivity, presumably related to the lower homogeneity of their sample and treatment approaches. Also opposed to the prediction of network theory [7], and not consistent with Smith et al. [17], network connectivity did not predict remission from binge eating at posttreatment or at 6-month follow-up. Thus, network connectivity was not revealed as an indicator of vulnerability and relapse across 6 months of follow-up. Further research is warranted to address the predictive value of network connectivity in the long term.

Descriptively, changes in network structure were mostly consistent with the therapeutic concept of CBT [20,45, 46], facilitating connections of core symptoms including binge eating and shape concern with their antecedents and consequences, thereby leading to simultaneous improvements among symptoms. Therefore, our study's increased network connectivity may not represent a greater psychopathology but rather a therapeutic gain in itself, likely reflecting an enhanced patient understanding of the interrelated nature of psychopathology. These conclusions require confirmation by future research. Of note, a supplementary analysis did not confirm a predictive value of the most central symptoms, as opposed to previous research on mixed eating disorders and obesity [38, 47, 48].

Considering strengths and limitations, our study was based on a large, multicenter RCT [18] using standardized CBT treatment protocols and validated interview- and questionnaire-based assessments through 6 months of follow-up. The cross-sectional network analyses replicated previous findings, lending support to the validity of the results. We had at least 3 times the observations as the number of parameters [36], and thus the sample size sufficed for the analyses and bootstrapping confirmed the ro- 
bustness of the results. Limitations are that the sample size precluded analyses by treatment so that it was not possible to explore psychopathological networks as moderators. Relatedly, we focused on aggregated scores and not on single indicators as done in some [16, 49, 50] but not all [13] network studies in eating disorders. We selected scales to optimally represent the clinical presentation of BED as reflected in treatment outcome research in this disorder [2], with a focus on maintaining factors postulated by the cognitive-behavioral model $[20,45,46]$ and by recent neuroscientifically informed conceptualizations [51].

Because of major differences between our study's results on network connectivity and treatment-related predictions of network theory, further elucidation of the pathological versus healthful nature of networks is necessary. Clarification is warranted regarding to which degree networks based on patient-reported symptoms reflect his/her understanding not only of the symptoms but also of the interrelations between them. Greater inclusion of objectively measured data [12] could represent a viable approach to address this issue. In addition, network analyses on the temporal succession of symptoms over treatment, using intensive longitudinal designs with multiple assessments [52], could help to elucidate causes and consequences of specific symptoms within dynamic networks of BED and the effects of interventions.

\section{Acknowledgement}

We thank Anne Brauhardt and Frauke Schmidt for their support in the conduct of this study, and Jamie L. Manwaring, Frederike Obereigner, and Josefine Kappe for their editorial support.

\section{Statement of Ethics}

This research was conducted ethically in accordance with the World Medical Association Declaration of Helsinki. Ethical approval was granted by the Ethics Committee of the University of Erlangen (reference No. 4081), based on which approval was granted by site-specific institutional review boards. Informed written consent was obtained at the outset of this study.

\section{Conflict of Interest Statement}

Dr. Hilbert reports giving lectures and workshops on the psychotherapy of BED and obesity; receiving honoraria as an editor with Springer, as a reviewer with Mercator Research Center Ruhr and Oxford University Press, and as a consultant with WeightWatchers and GlobalData; and publishing books with Hogrefe. Dr. Herpertz reports giving lectures and receiving travel grants from

Berlin Chemie, Lilly, and Sanofi and authoring books and articles on diabetes, obesity, and eating disorders published by Springer and Thieme. Dr. Zipfel reports authoring books and articles on psychotherapy of eating disorders published by Thieme, Springer, Routledge, and Elsevier. Dr. Tuschen-Caffier reports giving lectures and workshops on the psychotherapy of eating disorders and authoring books and articles on eating disorders published by Beltz, DGVT, Huber, Hogrefe, Kohlhammer, Psychosozial-Verlag, and Wiley. Dr. Friederich reports giving lectures and workshops on eating disorders and authoring books and articles on eating disorders published by Springer, Elsevier, Routledge, and Hogrefe. Dr. Mayr reports authoring articles on statistical methods and eating disorders published by Thieme, Springer, Wiley, and Elsevier; authoring books published by Springer; and giving workshops on statistics for Siemens Healthcare. Dr. de Zwaan reports serving on advisory boards, giving lectures, and receiving travel grants from Novo Nordisk and Danone and authoring books and articles on psychotherapy of eating disorders published by Thieme, Springer, Routledge, and Elsevier. No other disclosures are reported.

\section{Funding Sources}

This work was supported by the German Federal Ministry of Education and Research (BMBF; 01GV0601). Dr. Hilbert was funded by BMBF grant 01EO1501. The sponsors had no involvement in the study design; in the collection, analysis, and interpretation of data; in the writing of this report; or in the decision to submit this article for publication.

\section{Author Contributions}

Study concept and design: Dr. Hilbert, Dr. Mayr, and Dr. de Zwaan. Obtainment of funding: Dr. de Zwaan and Dr. Hilbert. Data analysis: Dr. Mayr. Interpretation of data and drafting of this paper: Dr. Hilbert, Dr. Mayr, and Dr. de Zwaan. Acquisition of data, critical revision of this paper for important intellectual content: Dr. Hilbert, Dr. Herpertz, Dr. Zipfel, Dr. Tuschen-Caffier, Dr. Friederich, Dr. Mayr, and Dr. de Zwaan

References

Psychother Psychosom 2020;89:379-385 DOI: $10.1159 / 000509458$
1 American Psychiatric Association. Diagnostic and statistical manual of mental disorders. 5th ed. Arlington: American Psychiatric Association; 2013.

2 Hilbert A, Petroff D, Herpertz S, Pietrowsky R, Tuschen-Caffier B, Vocks S, et al. Metaanalysis of the efficacy of psychological and medical treatments for binge-eating disorder. J Consult Clin Psychol. 2019 Jan;87(1):91105.

3 Linardon J, Wade TD, de la Piedad Garcia X, Brennan L. The efficacy of cognitive-behavioral therapy for eating disorders: A systematic review and meta-analysis. J Consult Clin Psychol. 2017 Nov;85(11):1080-94. 
4 Linardon J, de la Piedad Garcia X, Brennan L. Predictors, moderators, and mediators of treatment outcome following manualised cognitive-behavioural therapy for eating disorders: a systematic review. Eur Eat Disord Rev. 2017 Jan;25(1):3-12.

5 Borsboom D, Cramer AO. Network analysis: an integrative approach to the structure of psychopathology. Annu Rev Clin Psychol. 2013;9(1):91-121.

6 Borsboom D. A network theory of mental disorders. World Psychiatry. 2017 Feb;16(1):513.

7 van Borkulo C, Boschloo L, Borsboom D, Penninx BW, Waldorp LJ, Schoevers RA. Association of symptom network structure with the course of depression. JAMA Psychiatry. 2015 Dec;72(12):1219-26.

8 Beard C, Millner AJ, Forgeard MJ, Fried EI, Hsu KJ, Treadway MT, et al. Network analysis of depression and anxiety symptom relationships in a psychiatric sample. Psychol Med. 2016 Dec;46(16):3359-69.

9 Madhoo M, Levine SZ. Network analysis of the quick inventory of depressive symptomatology: reanalysis of the STAR*D clinical trial. Eur Neuropsychopharmacol. 2016 Nov; 26(11):1768-74.

10 Schweren L, van Borkulo CD, Fried E, Goodyer IM. Assessment of symptom network density as a prognostic marker of treatment response in adolescent depression. JAMA Psychiatry. 2018 Jan;75(1):98-100.

11 Fried EI, van Borkulo CD, Cramer AO, Boschloo L, Schoevers RA, Borsboom D. Mental disorders as networks of problems: a review of recent insights. Soc Psychiatry Psychiatr Epidemiol. 2017 Jan;52(1):1-10.

12 Contreras A, Nieto I, Valiente C, Espinosa R, Vazquez C. The study of psychopathology from the network analysis perspective: a systematic review. Psychother Psychosom. 2019; 88(2):71-83.

13 Levinson CA, Vanzhula IA, Brosof LC, Forbush K. Network analysis as an alternative approach to conceptualizing eating disorders: implications for research and treatment. Curr Psychiatry Rep. 2018 Aug;20(9): 67.

14 Smith KE, Crosby RD, Wonderlich SA, Forbush KT, Mason TB, Moessner M. Network analysis: an innovative framework for understanding eating disorder psychopathology. Int J Eat Disord. 2018 Mar;51(3):21422.

15 Wang SB, Jones PJ, Dreier M, Elliott H, Grilo $\mathrm{CM}$. Core psychopathology of treatmentseeking patients with binge-eating disorder: a network analysis investigation. Psychol Med. 2019 Aug;49(11):1923-8.

16 Solmi M, Collantoni E, Meneguzzo P, Degortes D, Tenconi E, Favaro A. Network analysis of specific psychopathology and psychiatric symptoms in patients with eating disorders. Int J Eat Disord. 2018 Jul; 51(7):680-92.
17 Smith KE, Mason TB, Crosby RD, Cao L, Leonard RC, Wetterneck CT, et al. A comparative network analysis of eating disorder psychopathology and co-occurring depression and anxiety symptoms before and after treatment. Psychol Med. 2019 Jan;49(2):31424.

18 de Zwaan M, Herpertz S, Zipfel S, Svaldi J, Friederich HC, Schmidt F, et al. Effect of internet-based guided self-help vs individual face-to-face treatment on full or subsyndromal binge eating disorder in overweight or obese patients: the INTERBED randomized clinical trial. JAMA Psychiatry. 2017 Oct; 74(10):987-95.

19 American Psychiatric Association. Diagnostic and statistical manual of mental disorders. 4th ed., text revision. Arlington: American Psychiatric Association; 2000.

20 Hilbert A, Tuschen-Caffier B. Essanfälle und Adipositas. Ein Manual zur kognitiv-behavioralen Therapie der Binge-Eating-Störung. Göttingen: Hogrefe; 2010.

21 Carrard I, Crépin C, Rouget P, Lam T, Golay A, Van der Linden M. Randomised controlled trial of a guided self-help treatment on the Internet for binge eating disorder. Behav Res Ther. 2011 Aug;49(8):482-91.

22 Fairburn CG, Cooper Z. The Eating Disorder Examination. In: Fairburn CG, Wilson GT, editors. Binge eating: nature, assessment, and treatment. New York: Guilford Press; 1993. pp. 317-56.

23 Hilbert A, Tuschen-Caffier B. Eating Disorder Examination: deutschsprachige Übersetzung (Bd. 01, 2. Auflage). Tübingen: dgvtVerlag; 2016.

24 Van Strien T, Rookus MA, Bergers GP, Frijters JE, Defares PB. Life events, emotional eating and change in body mass index. Int J Obes. 1986;10(1):29-35.

25 Grunert SC. Ein Inventar zur Erfassung von Selbstaussagen zum Ernährungsverhalten. Diagnostica. 1989;35(2):167-79.

26 Bohn K, Doll HA, Cooper Z, O’Connor M, Palmer RL, Fairburn CG. The measurement of impairment due to eating disorder psychopathology. Behav Res Ther. 2008 Oct;46(10): 1105-10.

27 Beck AT, Steer RA, Brown GK. Manual for the Beck Depression Inventory-II. San Antonio (TX): Psychological Corporation; 1996.

28 Hautzinger M, Keller F, Kühner C. Beck Depression Inventar II (BDI II). Frankfurt: Harcourt Test Service; 2006.

29 Rosenberg M. Society and the adolescent self-image. Princeton (NJ): Princeton University Press; 1965. https://doi.org/10.1515/ 9781400876136.

30 Von Collani G, Herzberg PY. Eine revidierte Fassung der deutschsprachigen Skala zum Selbstwertgefühl von Rosenberg. Z Differ Diagn Psychol. 2003;24(1):3-7.
31 Carver CS, White TL. Behavioral inhibition, behavioral activation, and affective responses to impending reward and punishment: the BIS/BAS Scales. J Pers Soc Psychol. 1994; 67(2):319-33.

32 Strobel A, Beauducel A, Debener S, Brocke B. Eine deutschsprachige Version des BIS/BASFragebogens von Carver und White. Z Differ Diagn Psychol. 2001;22(3):216-27.

33 Golino HF, Epskamp S. Exploratory graph analysis: A new approach for estimating the number of dimensions in psychological research. PLoS One. 2017 Jun;12(6):e0174035.

34 Golino H, Shi D, Christensen AP, Garrido LE, Nieto MD, Sadana R, et al. Investigating the performance of exploratory graph analysis and traditional techniques to identify the number of latent factors: A simulation and tutorial. Psychol Methods. 2020 Jun;25(3):292-320.

35 Epskamp S, Fried EI. A tutorial on regularized partial correlation networks. Psychol Methods. 2018 Dec;23(4):617-34.

36 Epskamp S, Borsboom D, Fried EI. Estimating psychological networks and their accuracy: A tutorial paper. Behav Res Methods. 2018 Feb;50(1):195-212.

37 van Borkulo C, Boschloo L, Kossakowski JJ, Tio P, Schoevers RA, Borsboom D, et al. Comparing network structures on three aspects: a permutation test. 2017. Available from: https://doi.org/10.13140/RG.2.2.29455. 38569.

38 Monteleone AM, Cascino G, Solmi M, Pirozzi $\mathrm{R}$, Tolone S, Terracciano G, et al. A network analysis of psychological, personality and eating characteristics of people seeking bariatric surgery: identification of key variables and their prognostic value. J Psychosom Res. 2019 May;120:81-9.

39 Forrest LN, Sarfan LD, Ortiz SN, Brown TA, Smith AR. Bridging eating disorder symptoms and trait anxiety in patients with eating disorders: A network approach. Int J Eat Disord. 2019 Jun;52(6):701-11.

40 Meier M, Kossakowski JJ, Jones PJ, Kay B, Riemann BC, McNally RJ. Obsessive-compulsive symptoms in eating disorders: A network investigation. Int J Eat Disord. 2020 Mar;53(3):362-71.

41 Christian C, Perko VL, Vanzhula IA, Tregarthen JP, Forbush KT, Levinson CA. Eating disorder core symptoms and symptom pathways across developmental stages: A network analysis. J Abnorm Psychol. 2020 Feb; 129(2):177-90.

42 Calugi S, Dalle Grave R. Psychological features in obesity: A network analysis. Int J Eat Disord. 2020 Feb;53(2):248-55.

43 Bringmann LF, Eronen MI. Don't blame the model: reconsidering the network approach to psychopathology. Psychol Rev. 2018 Jul; 125(4):606-15

44 Fried EI, Cramer AO. Moving forward: challenges and directions for psychopathological network theory and methodology. Perspect Psychol Sci. 2017 Nov;12(6):999-1020. 
45 Fairburn CG, Cooper Z, Shafran R. Cognitive behaviour therapy for eating disorders: a "transdiagnostic" theory and treatment. Behav Res Ther. 2003 May;41(5):509-28.

46 Murphy R, Straebler S, Cooper Z, Fairburn CG. Cognitive behavioral therapy for eating disorders. Psychiatr Clin North Am. 2010 Sep;33(3):611-27.

47 Olatunji BO, Levinson C, Calebs B. A network analysis of eating disorder symptoms and characteristics in an inpatient sample. Psychiatry Res. 2018 Apr;262:270-81.
48 Brown TA, Vanzhula IA, Reilly EE, Levinson CA, Berner LA, Krueger A, et al. Body mistrust bridges interoceptive awareness and eating disorder symptoms. J Abnorm Psychol. 2020 Mar. doi: 10.1037/abn0000516.

49 DuBois RH, Rodgers RF, Franko DL, Eddy KT, Thomas JJ. A network analysis investigation of the cognitive-behavioral theory of eating disorders. Behav Res Ther. 2017 Oct;97:213-21.

50 De Paoli T, Fuller-Tyszkiewicz M, Huang C, Krug I. A network analysis of borderline personality disorder symptoms and disordered eating. J Clin Psychol. 2020 Apr;76(4):787800 .
51 Kober H, Boswell RG. Potential psychological \& neural mechanisms in binge eating disorder: implications for treatment. Clin Psychol Rev. 2018 Mar;60:32-44.

52 Levinson CA, Vanzhula I, Brosof LC. Longitudinal and personalized networks of eating disorder cognitions and behaviors: targets for precision intervention a proof of concept study. Int J Eat Disord. 2018 Nov;51(11): 1233-43. 